\title{
ON APPROXIMATION IN THE BERS SPACES
}

\author{
CHARLES $\mathrm{K}$. CHUI
}

ABstract. Let $D$ be a Jordan domain in the complex plane with rectifiable boundary $C$. Let $A_{q}(D)$ denote the Bers space with norm \|\|$_{a}$. We prove that if $f \in A_{q}(D), 2<q<\infty$, then there exist functions $s_{n}(z)=\sum_{k=1}^{n} 1 /\left(z-z_{n, k}\right), \quad z_{n, k} \in C$ for $k=1, \cdots, n$, such that $\left\|s_{n}-f\right\|_{q} \rightarrow 0$. This result does not hold for $1<q \leqq 2$ even when $D$ is a disc.

1. Introduction and results. Let $D$ be a bounded Jordan domain in the complex plane with boundary $C$. For $1<q<\infty$, let $A_{q}(D)$ denote the Bers space, that is, the Banach space of holomorphic functions $f$ in $D$ with

$$
\|f\|_{q}=\iint_{D}|f(z)| \lambda_{D}^{2-q}(z) d x d y<\infty,
$$

where $\lambda_{D}(z)$ is the Poincare metric for $D$. Polynomial approximations in the Bers spaces have been considered by various authors. In the case $q \geqq 2$, Bers [2] and Knopp [5] proved that the polynomials are dense in $A_{q}(D)$. Recently, Metzger and Sheingorn [10] proved the polynomial density result for $q>1$ if $D$ is a Smirnov domain, and Metzger [9] proved this result for $q>3 / 2$ if the boundary curve $C$ is rectifiable. In the following, we will consider the approximation problem by the functions

$$
s_{n}(z)=\sum_{k=1}^{n} \frac{1}{z-z_{n, k}}, \quad z_{n, k} \in C,
$$

$k=1, \cdots, n$. The complex conjugate of $s_{n}(z)$ represents the gravitational (or electrostatic) field at the point $z$ due to unit masses (or charges) at the points $z_{n, k}$ (cf. [7]). Korevaar [6] proved that if $f$ is holomorphic in $D$, then there exist functions $s_{n}$ as in (2) which approximate $f$ uniformly on each compact subset of $D$. However, recently Newman [11] proved that if $D$ is the open unit disc and $\left|z_{n, k}\right|=1, k=1, \cdots, n$, then $\left\|s_{n}\right\|_{2} \geqq \pi / 18$ for all $n$. Hence, we cannot, in general, approximate in the spaces $A_{q}(D)$ by

Received by the editors July 19, 1972 and, in revised form, January 8, 1973.

AMS (MOS) subject classifications (1970). Primary 30A82.

Key words and phrases. Bers spaces, approximation, Jordan domain, gravitational field, unit masses.

(c) American Mathematical Society 1973 
the functions $s_{n}$ for $1<q \leqq 2$, when $D$ is an open disc. For $q>2$, we have the following:

THEOREM. Let $D$ be a Jordan domain with rectifiable boundary curve $C$. Let $f \in A_{q}(D)$ where $2<q<\infty$. Then there exist functions $s_{n}$ as in (2) such that $\left\|s_{n}-f\right\|_{q} \rightarrow 0$.

2. Proof of the Theorem. Let $\phi$ be a conformal map from the exterior of the unit circle onto the exterior of $C$ such that $\phi(\infty)=\infty$ and such that the continuous extension to the closure, which we also denote by $\phi$, maps the point 1 to a point $z_{0} \in C$. Let $\psi(t)=\phi\left(e^{i 2 \pi t}\right)$, and denote the diameter of $D$ by $d$ and the length of $C$ by $l$. For $2<q<\infty$, we have

$$
\left\|\int_{C} \frac{|d \zeta|}{|z-\zeta|^{2}}\right\|_{q}<\frac{2 \pi l}{q-2}(4 d)^{a-2}
$$

and

$$
\left\|\int_{0}^{1} \frac{d t}{|z-\psi(t)|}\right\|<\frac{2 \pi}{q-1} 4^{q-2} d^{q-1} .
$$

Indeed, by the Koebe- $\frac{1}{4}$ Theorem (cf. [4]) we have $\lambda_{D}(z) \operatorname{dist}(z, C) \geqq \frac{1}{4}$ for all $z \in D$ (cf. [2]), so that for each $\zeta \in C$, we have

$$
\begin{aligned}
\iint_{D} \frac{\lambda_{D}^{2-q}(z)}{|z-\zeta|^{2}} d x d y & \leqq 4^{a-2} \iint_{D}|z-\zeta|^{a-4} d x d y \\
& <4^{a-2} \iint_{|z-\zeta|<d}|z-\zeta|^{q-4} d x d y \\
& =2 \pi 4^{a-2} \int_{0}^{d} r^{a-3} d r=\frac{2 \pi}{q-2}(4 d)^{a-2}
\end{aligned}
$$

and, hence,

$$
\int_{C}\left\{\iint_{D} \frac{\lambda_{D}^{2-q}(z)}{|z-\zeta|^{2}} d x d y\right\}|d \zeta|<\frac{2 \pi l}{q-2}(4 d)^{a-2},
$$

proving (3). (4) can be proved similarly.

Now suppose that $f \in A_{q}(D)$. Since the polynomials are dense in $A_{q}(D)$ (cf [2], [5]), we can assume that $f$ is an entire function. From a representation formula of Mac Lane [8], we can write

$$
f(z)=\int_{0}^{1} \frac{\mu(t) d t}{z-\psi(t)}
$$


for each $z \in D$, where $\mu(t)$ is a real-valued analytic function on $[0,1]$. Let

$$
f_{n}(z)=\frac{1}{n} \sum_{k=1}^{n} \frac{\mu(k / n)}{z-\psi(k / n)},
$$

and for a real $x$ we denote, as usual, the largest integer no greater than $x$ by $[x]$. Then

$$
\begin{aligned}
f(z)-f_{n}(z) & =\frac{1}{n} \int_{0}^{1} \frac{\mu(t)}{z-\psi(t)} d\{n t-[n t]\} \\
& =-\frac{1}{n} \int_{0}^{1} \frac{\{n t-[n t]\}}{\{z-\psi(t)\}^{2}}\left\{\mu^{\prime}(t)(z-\psi(t))+\mu(t) \psi^{\prime}(t)\right\} d t
\end{aligned}
$$

so that

$$
\left\|f-f_{n}\right\|_{q} \leqq \frac{1}{n}\left\|\int_{0}^{1} \frac{\mu^{\prime}(t) d t}{|z-\psi(t)|}\right\|_{q}+\frac{c}{n}\left\|\int_{c \mid} \frac{|d \zeta|}{|z-\zeta|^{2}}\right\|_{a},
$$

where $c$ is an upper bound of $|\mu(t)|$ on $[0,1]$. By (3) and (4), we conclude that $\left\|f-f_{n}\right\|_{q}=O(1 / n)$. Hence, we can assume that $f(z)=\alpha /\left(z-z_{1}\right)$ where $z_{1} \in C$ and $-1<\alpha<1$. Actually, the above proof is independent of the point $z_{0}$, and for convenience, we can take $z_{0}=z_{1}$.

Now, for each $z \in D$, we have

$$
\int_{0}^{2 \pi} \frac{d t}{z-\phi\left(e^{i t}\right)}=\frac{2 \pi}{z-\phi(\infty)}=0 .
$$

Modifying a construction in [3], we let

$$
\begin{gathered}
t_{n, 0}=0, \quad t_{n, 1}=\frac{(2-\alpha) \pi}{n+1-\alpha}, \\
t_{n, 2}=\frac{(2-\alpha) \pi+2 \pi}{n+1-\alpha}, \cdots, t_{n, n}=\frac{(2-\alpha) \pi+2(n-1) \pi}{n+1-\alpha} ;
\end{gathered}
$$

and $z_{n, k}=\phi\left(e^{i t_{n, k}}\right), k=0, \cdots, n$. Let $\mu_{n}(t)$ be the step function with discontinuities only at the $t_{n, k}$ such that

$$
u_{n}\left(t_{n, k}^{+}\right)-u_{n}\left(t_{n, k}^{-}\right)=1
$$

for $k=1, \cdots, n, u_{n}\left(0^{+}\right)=(1-\alpha) / 2$ and $u_{n}\left(2 \pi^{-}\right)=n+(1-\alpha) / 2$. We also take $u_{n}(0)=0, u_{n}(2 \pi)=n+1-\alpha$ and

$$
u_{n}\left(t_{n, k}\right)=\left[u_{n}\left(t_{n, k}^{+}\right)+u_{n}\left(t_{n, k}^{-}\right)\right] / 2
$$

for $k=1, \cdots, n$. Then it is clear that, for each $z \in D$,

$$
\sum_{k=0}^{n} \frac{1}{z-z_{n, k}}-\frac{\alpha}{z-z_{0}}=\int_{0}^{2 \pi} \frac{d u_{n}(t)}{z-\phi\left(e^{i t}\right)} .
$$


Let $v_{n}(t)=u_{n}(t)-(n+1-\alpha) t / 2 \pi$. By (5) and (6) we have

$$
\sum_{k=0}^{n} \frac{1}{z-z_{n, k}}-\frac{\alpha}{z-z_{0}}=\int_{0}^{2 \pi} \frac{d v_{n}(t)}{z-\phi\left(e^{i t}\right)} .
$$

But by the construction of $u_{n}$ and $v_{n}$, it is clear that $v_{n}(0)=v_{n}(2 \pi)=$ $v_{n}\left(t_{n, k}\right)=0$ for $k=1, \cdots, n$; and hence, it is not difficult to see that

$$
\sup _{0 \leqq t \leqq 2 \pi}\left|v_{n}(t)\right| \leqq \max \left\{\frac{1}{2}, \frac{1-\alpha}{2}\right\} \leqq 1 .
$$

Let $E$ be any subset of $D$. Integrating (7) by parts, we obtain, by using (8), that

$$
\begin{aligned}
\iint_{E} \mid \sum_{k=0}^{n} & \frac{1}{z-z_{n, k}}-\frac{\alpha}{z-z_{0}} \mid \lambda_{D}^{2-q}(z) d x d y \\
& \leqq \iint_{E} \int_{0}^{2 \pi}\left|\frac{d \phi\left(e^{i t}\right)}{\left(z-\phi\left(e^{i t}\right)\right)^{2}}\right| \lambda_{D}^{2-q}(z) d x d y \\
& \leqq \iint_{E}\left\{\int_{C} \frac{|d \zeta|}{|z-\zeta|^{2}} \mid \lambda_{D}^{2-q}(z) d x d y=\iiint \int_{E} \frac{\lambda_{D}^{2-q}(z)}{|z-\zeta|^{2}} d x d y\right\}|d \zeta| .
\end{aligned}
$$

Let $\varepsilon>0$ be arbitrarily chosen. By (3), we can find a compact subset $K=$ $K_{\varepsilon}$ of $D$ such that

$$
\iint_{C}\left\{\int_{D \backslash K} \frac{\lambda_{D}^{2-\alpha}(\dot{z})}{|z-\zeta|^{2}} d x d y\right\}|d \zeta|<\varepsilon
$$

Hence, for all $n$, we have, from (9),

$$
\iint_{D \backslash K}\left|\sum_{k=0}^{n} \frac{1}{z-z_{n, k}}-\frac{\alpha}{z-z_{0}}\right| \lambda_{D}^{2-a}(z) d x d y<\varepsilon .
$$

On the other hand, since $C$ is rectifiable, it is well known that $\phi^{\prime}\left(e^{i t}\right)$ : is Lebesgue integrable on $[0,2 \pi]$. Hence, it can be shown, by using (8) and a proof similar to that of the Riemann-Lebesgue lemma, that

$$
\int_{0}^{2 \pi} v_{n}(t) \frac{\phi^{\prime}\left(e^{i t}\right) e^{i t}}{\left(z-\phi\left(e^{i t}\right)\right)^{2}} d t \rightarrow 0
$$

uniformly on each compact subset of $D$. (In doing this, we note that $v_{n}(t)$ has the behavior similar to cos $n t$ as in the proof of the Riemann-Lebesgue 
lemma.) Hence, by (7) we have

$$
\sum_{k=0}^{n} \frac{1}{z-z_{n, k}} \rightarrow \frac{\alpha}{z-z_{0}}
$$

uniformly on the compact set $K$. By combining this with (10), we have completed the proof of the theorem.

\section{REFERENCES}

1. L. Bers, An approximation theorem, J. Analyse Math. 14 (1965), 1-4. MR 31 \#2545.

2. - A nonstandard integral equation with applications to quasiconformal mappings, Acta Math. 116 (1966), 113-134. MR 33 \#273.

3. C. K. Chui, Bounded approximation by polynomials whose zeros lie on a circle, Trans. Amer. Math. Soc. 138 (1969), 171-182. MR 38 \#6076.

4. E. Hille, Analytic function theory. Vol. II, Introduction to Higher Math., Ginn, Boston, Mass., 1962. MR 34 \#1490.

5. M. I. Knopp, A corona theorem for automorphic forms and related results, Amer. J. Math. 91 (1969), 599-618. MR 40 \#4450.

6. J. Korevaar, Asymptotically neutral distributions of electrons and polynomial approximation. Ann. of Math. (2) 80 (1964), 403-410. MR 29 \#6031.

7. - Limits of polynomials whose zeros lie in a given set, Proc. Sympos. Pure Math., vol. 11, Amer. Math. Soc., Providence, R.I., 1968, pp. 261-272. MR 38 \#2282.

8. G. R. Mac Lane, Polynomials with zeros on a rectifiable Jordan curve, Duke Math.

J. 16 (1949), 461-477. MR 11, 20.

9. T. A. Metzger, On polynomial approximation in $A_{q}(D)$, Proc. Amer. Math. Soc. 37 (1973), 468-470.

10. T. A. Metzger and M. Sheingorn, Polynomial approximations in the Bers spaces (to appear).

11. D. J. Newman, $A$ lower bound for an area integral, Amer. Math. Monthly 79 (1972), 1015-1016.

Department of Mathematics, Texas a\&M University, College Station, TEXAS 77843 